\title{
SHOULD NATIONAL INSTITUTE FOR CLINICAL EXCELLENCE (NICE) GUIDELINES REMOVE URINE CYTOLOGY AS A SUGGESTED ADJUNCT IN SUSPECTED BLADDER CANCER (HAEMATURIA) INVESTIGATIONS?
}

Madeline Moore $^{1 *}$, Andrew Robinson ${ }^{2 *}$, Mark O Kitchen ${ }^{1,2}$, Lyndon Gommersall ${ }^{1,2}$

${ }^{1}$ Urology Department, University Hospitals of North Midlands NHS Trust, Staffordshire, UK.

${ }^{2}$ School of Medicine, Keele University, Staffordshire, UK

*joint first authors (both contributed equally)

\section{Corresponding author: m.o.kitchen@keele.ac.uk}

\begin{abstract}
\section{Background}

Despite National Institute for Clinical Excellence (NICE) guidelines suggesting the use of urine cytology (UC) for the diagnosis of bladder cancer, its use is variable. Reasons for this include sub-optimal sensitivity, financial cost, availability of alternative tests, and uncertainty over interpretation of results. Anecdotally, however, suspicious or malignant UC when other investigations are normal, occasionally leads to a cancer diagnosis. Therefore, we retrospectively assessed a cohort of our haematuria patients to determine the value of UC in cancer diagnosis and the clinical significance of atypical UC (graded as C3).
\end{abstract}

\section{Patients and methods}

We identified 3018 patients with haematuria referred on the suspected cancer pathway ("two-week wait") in 2015. We retrospectively analysed clinical, demographic, and follow-up/outcome data in a random cohort of 500 cases.

\section{Results}

Median follow up was 58 months. Urological malignancy was diagnosed in 61/500 patients; all were identified by cystoscopy or imaging, i.e., irrespective of UC result. No cases of atypical UC re-presented with a 'missed' cancer diagnosis within the five-year follow-up period. However, suspicious and malignant cytology was associated with high-grade/aggressive tumours and subsequent tumour recurrence.

\section{Conclusion}

Urine cytology did not identify any cancers that were not already found by imaging or cystoscopy. Atypical $\mathrm{UC}$ in the presence of negative haematuria investigations does not appear to be associated with malignancy, and therefore should not alter patient management nor prompt further investigation. Suspicious and malignant UC was associated with higher risk cancers and could therefore be used to prioritise waiting lists for transurethral resection of bladder tumour (TURBT), however, it is unclear whether this might benefit patient outcomes. We conclude therefore that UC has no role in haematuria investigations.

Key words: haematuria, suspected cancer referral, urine cytology

J Endolum Endourol Vol 4(3):e1-e7; October 12, 2021. 


\section{INTRODUCTION}

Current National Institute for Clinical Excellence (NICE) guidelines suggest that assessment of patients with haematuria, should comprise urine microbiological culture and protein:creatinine ratio, blood tests including estimated glomerular filtration rate (eGFR), upper tract imaging, and direct visualisation of the bladder (flexible cystoscopy). ${ }^{1}$ In addition to these 'standard' investigations, NICE guidelines recommend using an adjunct diagnostic test in the context of suspected bladder cancer. These include narrow-band imaging, urine cytology, or another urinary biomarker. ${ }^{1}$ However, there is huge variation in practice across the UK between centres, and even within individual departments, as to how, when, and which test is used as an adjunct in bladder cancer diagnosis; furthermore, in some centres, none of the available adjunct investigations are used. ${ }^{2}$

Our University teaching hospital and tertiary cancer centre uses urine cytology (UC) as part of initial haematuria investigations. UC has variable sensitivity and specificity for bladder cancer $(28-100 \%$ and $83-99.7 \%$, respectively, ${ }^{3-5}$ ), dependent on tumour factors such as grade, stage, multifocality, and size (i.e., factors that might affect tumour cell exfoliation into urine). ${ }^{4} \mathrm{UC}$ is particularly sensitive for bladder carcinoma-in-situ (CIS), where, as a highly exfoliative but flat urothelial lesion, this clinically significant tumour type may be missed on flexible cystoscopy but detected by UC[5]. Our department favours UC given the high smoking rates and prevalent (but historic) environmental risk factors locally, such as tyre manufacture and the pottery industries. These factors likely contribute to a higher-than-average prevalence of high-risk non-muscle-invasive bladder cancer and CIS in our patients. ${ }^{6}$ Additionally, UC is a relatively inexpensive investigation (approximately $£ 70$ per test), is non-invasive, and can be quick to perform, albeit with the caveat that histopathologist review adds time and service demands. We use a standardised (Paris) urine cytology reporting system: $\mathrm{C} 1$ - insufficient for analyses, $\mathrm{C} 2$ - benign, $\mathrm{C} 3$ - atypical, $\mathrm{C} 4$ - suspicious, and $\mathrm{C} 5$ - malignant, which aids in risk stratification and can be easily used by allied health professionals, such as nurse specialists. ${ }^{7}$
Anecdotally, we noticed that a significant number of UC samples return as 'insufficient' or 'atypical.' This presents clinical diagnostic uncertainty, which has resulted in multiple case discussions at cancer multi-disciplinary team (MDT) meetings and numerous repeated cytology tests. This ultimately leads to delay in diagnosis, treatment, or discharge for patients (with resultant anxiety) and increases financial and service demands on already over-stretched services. Furthermore, in our experience, the repeat UC has seldom been 'upgraded' to suspicious (C4) or malignant (C5) and has rarely led to a cancer diagnosis that would not have been made without the cytology result. In view of this we retrospectively analysed our haematuria pathway patient's urine cytology results within the context of their suspected bladder cancer investigations, with the premise of understanding whether UC has any role as a diagnostic adjunct to routine haematuria investigations or whether it just causes clinical confusion, uncertainty, and leads to unnecessary investigations, procedures and burden on our service locally and the wider NHS.

\section{PATIENTS AND METHODS}

All patients referred to our University teaching hospital on the suspected bladder cancer ("two-week wait haematuria") pathway in 2015 were identified $(n=3018)$. A random sample of 500 cases (including patients with visible and non-visible haematuria) was selected for analyses. Patients were randomised using the 'random sort' function in Microsoft Excel (Microsoft Corporation, USA, v16.0, 2016).

Patients were excluded if they did not attend ('second stop') for further investigations $(n=6)$. Data collected included smoking status, occupational and environmental risk factors for urothelial cell carcinoma (UCC), comorbidities, and outcomes of haematuria investigations. As part of our local haematuria pathway, patients with VH underwent CT Urogram (unless specific contraindications such as poor renal function or contrast allergy), and patients with NVH routinely underwent ultrasound (in some cases, subsequent CTU was performed based upon US findings or patient risk factors for UCC)

Microsoft Excel and online MedCalc software ${ }^{8}$ were used for statistical analyses and multivariate analyses

J Endolum Endourol Vol 4(3):e1-e7; October 12, 2021.

This article is distributed under the terms of the Creative Commons Attribution-Non

Commercial 4.0 International License. (C) Moore et al. 
where appropriate $(\mathrm{p}<0.05$ considered significant), otherwise simple descriptive methods were used.

\section{RESULTS}

\section{Demographics}

Of the 500 cases evaluated, 294 (58.8\%) were female, and 206 (41.2\%) were male. The age range was $33-92$ years, median 67 . The follow-up ranged from 54 - 66 months, median 58. Thus, 302 (60.4\%) cases were referred for non-visible haematuria $(\mathrm{NVH})$ and 198 (39.6\%) for visible haematuria $(\mathrm{VH})$. Seventy-eight patients $(15.6 \%)$ were current or previous smokers, $34(6.8 \%)$ had potential occupational risk factors for UCC, and five $(1.0 \%)$ had a relevant family history of bladder cancer. The majority of referrals, 478 cases $(95.6 \%)$, were from Primary care; the remaining comprised $12(2.4 \%)$ from renal medicine outpatients, six $(1.2 \%)$ from urology outpatients, and four $(0.8 \%)$ from general medical inpatient admissions (all were still suspected cancer pathway referrals).

We identified a total of 61 urological malignancies $(12.2 \%$ of patients); four prostate $(0.8 \%$ total cohort), five upper tract (four renal tumours and one upper tract (renal pelvic) UCC, 1\%), and 52 bladder cancers (10.4)

\section{UC outcome}

Of the 500 urine cytology reports, 48 (9.6\%) were insufficient for analyses (C1), $302(60.4 \%)$ were benign (C2), 114 (22.8\%) were atypical (C3), 25 (5.0\%) were suspicious $(\mathrm{C} 4)$ and $21(4.2 \%)$ were malignant (C5). Of these cases, $2 \mathrm{C} 1,9 \mathrm{C} 2,12 \mathrm{C} 3,17 \mathrm{C} 4$ and $21 \mathrm{C} 5$ cases were associated with a cancer diagnosis, respectively (Figure 1.).

Of the 114 atypical $\mathrm{C} 3$ cytology results, eight (7.0\%) were brought to cancer multi-disciplinary team (MDT) meetings for case discussion and review, and $61(53.5 \%)$ were repeated (no apparent cause for the atypical result). Of the remaining $45 \mathrm{C} 3$ results, 12 patients had positive microbiological urine culture (e.coli and Kelbsiella species).

Of the 61 that were repeated, importantly, none returned as $\mathrm{C} 4$ or $\mathrm{C} 5$ (suspicious or malignant); 9 were $\mathrm{C} 1,29$ were $\mathrm{C} 2$, and 23 were $\mathrm{C} 3$. A further $16 \mathrm{UC}$ were performed a third time in patients that had $\mathrm{C} 3$ twice before; again, none returned as $\mathrm{C} 4$ or $\mathrm{C} 5$ on this repeat, and none were found to have Urological malignancy.

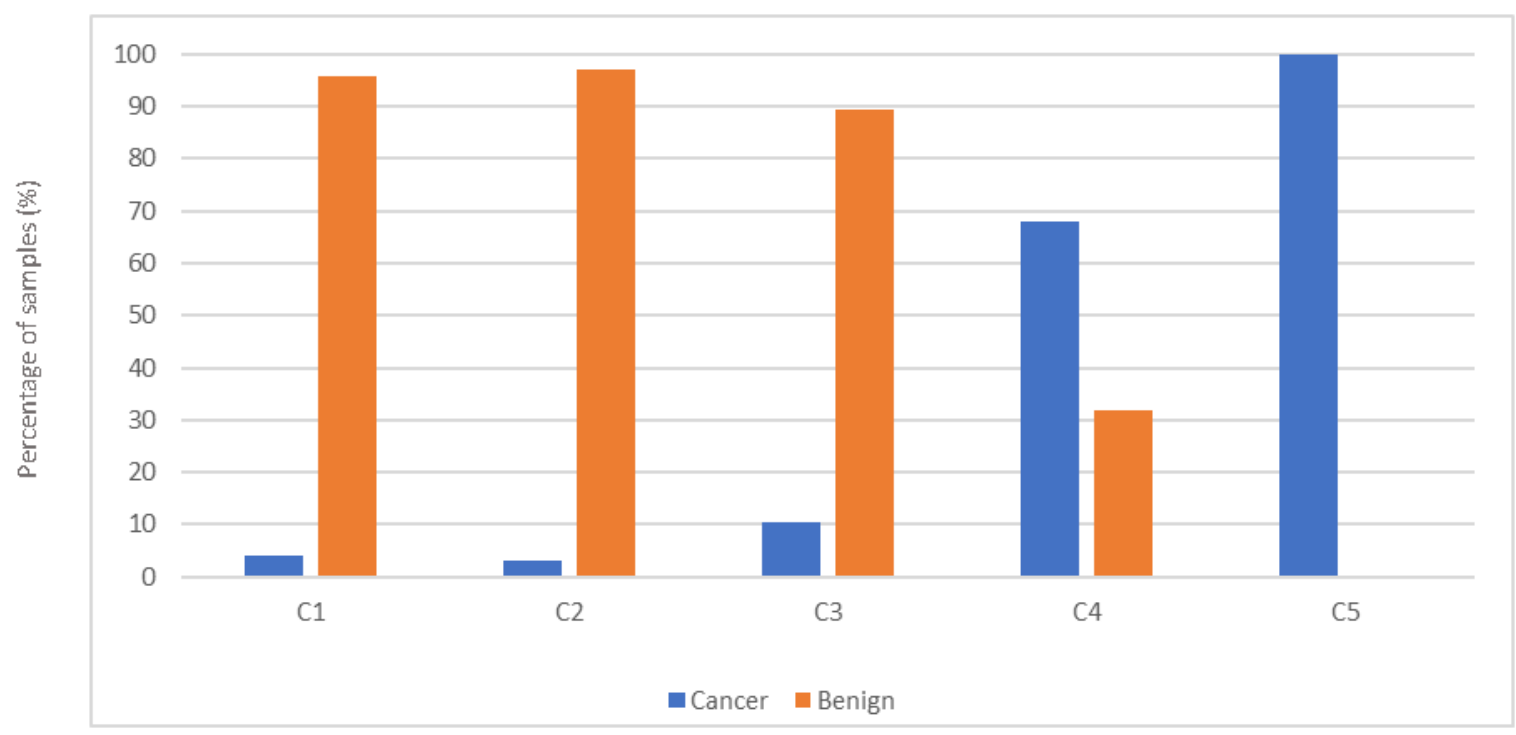

Figure 1. Percentage of cytology results associated with benign or cancer diagnosis Bar chart illustrating the percentage of each urine cytology grade (C1-C5) associated with cancer diagnosis (blue bar) or benign/non-cancer diagnosis (orange bar) at the conclusion of all haematuria investigations

J Endolum Endourol Vol 4(3):e1-e7; October 12, 2021.

This article is distributed under the terms of the Creative Commons Attribution-Non

Commercial 4.0 International License. (c) Moore et al. 
Identifying Silent Fungal Infections complicating Obstructive Uropathy

Table 1. Diagnostic Performance of Urine Cytology

\begin{tabular}{|l|c|c|c|c|}
\hline & Sensitivity & Specificity & Negative Predictive Value (NPV) & Positive Predictive Value (PPV) \\
\hline $\begin{array}{l}\text { C2 benign, C4 and } \\
\text { malignant }\end{array}$ & $80.0 \%$ & $97.3 \%$ & $65.4 \%$ & $98.7 \%$ \\
\hline $\begin{array}{l}\text { C2 } \text { and C3 benign, } \\
\text { Cnd C5 malignant }\end{array}$ & $63.2 \%$ & $99.2 \%$ & $82.6 \%$ & $97.7 \%$ \\
\hline
\end{tabular}

Table showing the sensitivity, specificity, negative and positive predictive values of 'positive' urine cytology as diagnostic for bladder or upper tract urothelial cancer, compared to 'negative' benign cystology; the first row considers only C2 cytology to be benign. The second row considers both $C 2$ and $C 3$ UC as benign/negative

Table 2. Multivariate Regression Analyses for Bladder and Upper Tract Urothelial Cancer Risk Factors

\begin{tabular}{|l|l|}
\hline Positive urine cytology & $\mathrm{P}<0.001$ \\
\hline Age $\geq 75$ & $\mathrm{P}=0.001$ \\
\hline Male gender & $\mathrm{P}=0.063$ \\
\hline Visible haematuria & $\mathrm{P}=0.012$ \\
\hline Current or previous smoker & $\mathrm{P}=0.097$ \\
\hline Occupational risk factor(s) & $\mathrm{P}=0.532$ \\
\hline
\end{tabular}

Table showing the outcome of multivariate regression analyses, identifying positive urine cytology, age $\geq 75$, and visible haematuria as risk factors significantly associated with bladder or upper tract urothelial cancer diagnosis in patients with haematuria. $P<0.05$ is considered statistically significant.

Of those who had a $\mathrm{C} 4$ or $\mathrm{C} 5$ result, $29 / 38$ (76.3\%) were associated with tumours that were ultimately high-risk (including locally advanced prostate, upper tract UCC, muscle-invasive bladder cancer, or high-risk non-muscle invasive bladder cancer). Of the eight patients with $\mathrm{C} 4 \mathrm{UC}$, no malignancy was found on routine haematuria investigations, and three underwent further investigation (two photodynamic diagnosis "PDD" cystoscopy, and one rigid cystoscopy with bilateral ureteroscopy upper tract UC sampling); all cases were negative/benign. The other five cases had their UC repeated, of which two remained $\mathrm{C} 4$ and were subject to MDT discussion but ultimately discharged. In these cases, urinary tract stone disease identified on imaging and microbiologically proven urinary tract infections were deemed to be the cause of the C4 UC. There was no recorded patient morbidity from any additional procedure undertaken because of abnormal UC.

\section{Sensitivity and specificity}

Using $\mathrm{C} 2$ as benign and classifying $\mathrm{C} 4$ and $\mathrm{C} 5$ as malignant, 'positive' UC had a sensitivity of $80.0 \%$ and specificity of $97.3 \%$, and positive and negative predictive values of $65.4 \%$ and $98.7 \%$, respectively, for bladder or upper tract cancer diagnosis in our cohort. Using $\mathrm{C} 2$ and $\mathrm{C} 3$ as benign, and $\mathrm{C} 4$ and $\mathrm{C} 5$ as malignant, positive UC had a sensitivity of $63.2 \%$ and specificity of $99.2 \%$, and positive and negative predictive values of $82.6 \%$ and $97.7 \%$, respectively, for bladder or upper tract cancer diagnosis (Table 1.).

\section{Regression analyses}

As shown in Table 2., visible haematuria, age equal to or greater than 75 , and suspicious and malignant cytology ( $\mathrm{C} 4$, and $\mathrm{C} 5$ respectively) were significantly associated with a bladder cancer diagnosis $(\mathrm{p}<0.05)$. Smoking status (current or ex-) and male gender were not significantly associated, but positively correlated with, cancer diagnosis and trended toward significance.

\section{Diagnostic relevance of $U C$}

Despite initial atypical (C3) or suspicious cytology (C4), leading to additional, and sometimes invasive investigations for the majority of cases, we did not find any positive UC where all other haematuria investigations were negative led to an initially 'missed' cancer diagnosis, i.e. all cancers were identified by imaging and/or flexible cystoscopy, and no cancers would have been missed if UC had not been performed.

\section{DISCUSSION}

Urine cytology has proven helpful in bladder and upper tract cancer surveillance, mainly where, for example, narrow-band imaging or photodynamic diagnosis cystoscopy are unavailable. In these cases, and in patients who have received pelvic irradiation or intravesical therapies, UC has an important role in differentiating non-malignant erythematous urothelium (identified commonly as "red patches" 
on surveillance cystoscopy) from malignant urothelium/tumour recurrence. ${ }^{5,9} \mathrm{UC}$ is beneficial if it was positive at initial presentation; in such cases, it can provide confidence to the clinician and reassurance to the patient during surveillance if it remains negative/ benign, or prompts further investigation if it becomes suspicious/malignant.

However, as an adjunct to diagnosis in suspected bladder cancer, the value of UC is less clear, mainly when it is performed on all haematuria patients, not just in those in whom bladder (or upper tract) cancer is suspected. Previous studies have suggested some benefit of UC in this context, albeit minimal, quoting figures of between $0.07 \%$ and $0.2 \%$ of patient cohorts where bladder cancers were missed by imaging and cystoscopy but suggested by positive UC. ${ }^{10,11}$ Therefore, although there is some evidence to suggest that there some 'missed' cancers are detected by UC, and anecdotal/experiential evidence to the same effect, similar to the recent DETECT I study cohort, ${ }^{2,4}$ we did not find this diagnostic benefit in our patients. Specifically, there were no instances of a positive UC result where other initial haematuria investigations were negative, and therefore no cancers were only detected by UC. We recognise, however, that as a retrospective single-centre study, our random cohort data may not be fully representative of the local population nor generalisable to the rest of the UK. In addition, we recognise the limitations that we have assumed on the basis of available clinic letters, MDT discussions and imaging, that patients have not been re-referred or otherwise diagnosed with any urological malignancy during the five-year follow-up period, however, the all-cause mortality rate in this predominantly elderly population, and the movements of patients out of the area, may affect the reliability of our data.

Interestingly, we found that the sensitivity and specificity for bladder cancer diagnosis, where $\mathrm{C} 2$ cytology is 'negative', and C4 and C5 are 'positive,' was slightly higher than some previous reports. ${ }^{2}$ This may reflect a chance finding in our random cohort but is more likely to reflect a combination of our higher-than-average prevalence of cancers, particularly high-risk tumours, within our local population and the experience and expertise of the histo/cytopathology consultants reporting the samples. ${ }^{12}$ The positive and negative predictive values were calculated based upon our cohort bladder and upper tract cancer prevalence. We advise caution in the interpretation of these values and their generalisability, given the variability in bladder cancer prevalence, the sporadic use of UC, and inter-user reporting variability and differences in classification systems used across the UK., ${ }^{2,13}$

Additionally, and although previous reports offer conflicting data regarding atypical urine cytology, ${ }^{14,15}$ we did not find any significant association of atypical (C3) UC with cancer in our cohort, and reassuringly, no patients who had C3 UC at presentation in 2015, represented with a 'missed' cancer within the five-year follow-up period. Furthermore, we found no increase in smoking status or presence of occupational or family risk factors in cases with C3 cytology (compared to other grades) that might suggest further investigation in these cases is warranted.

Although atypical UC was not associated with cancer, similarly to previous reports, our data highlighted the strong association of C4 and C5 cytology with high-risk tumours, and tumours that recurred over the follow-up period. ${ }^{9,14}$ One could therefore suggest that patients with $\mathrm{C} 4$ or $\mathrm{C} 5$ cytology could or should be prioritised for TURBT or nephroureterectomy; however, even in cases where we attempted this, waiting times for TURBT were seldom reduced by more than a week, and it is difficult to estimate any potential beneficial effect this may have had on the patient or oncological outcomes.

Whilst we support the use and recognise the clear benefits of performing UC for bladder and upper tract cancer surveillance, its use in haematuria investigations has frequently introduced diagnostic uncertainty and has primarily been disadvantageous in our experience. In particular, atypical C3, UC results have led to many repeated samples, discussions at cancer MDT, and additional invasive investigations (all negative within our representative cohort). This has introduced, in retrospect, unnecessary delays in patient management or reassurance and discharge, with resultant patient anxiety.

It is important to note also the volume of work that UC sample analyses represent for the histo/cytopathology department, particularly in the context of the current UK national workforce shortage in this specialty, with

J Endolum Endourol Vol 4(3):e1-e7; October 12, 2021.

This article is distributed under the terms of the Creative Commons Attribution-Non

Commercial 4.0 International License. (C) Moore et al. 
only $3 \%$ of laboratories being adequately staffed. ${ }^{16}$ The thousands of samples per year analysed and reported by histo/cytopathology consultants at our Trust has almost certainly contributed to the (understandable) delays in sample processing and increased waiting time for results that we observed in our study, with the 'knock-on' effects of delay in diagnosis, and again resultant patient anxiety, and inevitable cancer target breaches. One must likewise consider the significant financial implications that the uncertainty of UC introduces, primarily in the cost of repeated samples (approximately 15\% of all patients in our cohort), but also in the further invasive investigations, including the costs of general anaesthesia and theatre time. Perhaps more importantly, cancer target breach penalties may be incurred when patients remain on the tracked cancer pathway 'clock' and are not 'taken off' pending the results of these additional investigations. We estimate that $5-10 \%$ of our haematuria cases may risk target breaches due to the responsible clinician not actively removing the patient from the cancer pathway clock at the time of requesting a repeat sample. Therefore, it is difficult to estimate the potential cost savings of not performing UC routinely; however, our conservative estimate, based upon the number of UC samples analysed per year (excluding cancer target breach fines), is $£ 250,000$.

\section{CONCLUSION}

Where $\mathrm{UC}$ was performed as an adjunct to diagnosis in haematuria, it did not identify any cancers missed by imaging or flexible cystoscopy. However, it led to unnecessary repeated investigations, delay in patient reassurance and discharge, and the added finance of this repeat test. NICE should therefore remove UC from the suggested haematuria investigations, but we advocate its use in patients where bladder or upper tract tumours are found, given its important role in disease surveillance.

Furthermore, atypical (C3) urine cytology in the presence of negative initial haematuria investigations was not associated with malignancy or with 'missed' cancers during the five-year follow-up period, and therefore, we suggest it does not require repeating nor further investigation. On the other hand, suspicious and malignant $\mathrm{UC}, \mathrm{C} 4 / \mathrm{C} 5$, was associated with higher risk cancers and could therefore be used as a tool to aid prioritisation in waiting lists for TURBT.

\section{REFERENCES}

1. NICE. Bladder cancer: diagnosis and management of bladder cancer. BJU Int 2017;120:755-65.

2. Tan WS et al. Does urinary cytology have a role in haematuria investigations? BJU Int. 2019 Jan;123(1): 74-81.

3. Babjuk M et al. EAU Guidelines on Non-Muscle-invasive Urothelial Carcinoma of the Bladder: Update 2016. Eur Urol 2017 Mar;71(3):447-61.

4. Yafi FA, Brimo F, Steinberg J, et al. Prospective analysis of sensitivity and specificity of urinary cytology and other urinary biomarkers for bladder cancer. Urol Oncol 2015 Feb;33(2):66.e25-31.

5. Maas M, Bedke J, Stenzl A, Todenhöfer T. Can urinary biomarkers replace cystoscopy? World J Urol 2019; (37):1741-49.

6. IARC Working Group on the Evaluation of Carcinogenic Risks to Humans. Chemical Agents and Related Occupations. Lyon (FR): International Agency for Research on Cancer; 2012. (IARC Monographs on the Evaluation of Carcinogenic Risks to Humans, No. 100F.) OCCUPATIONAL EXPOSURES IN THE RUBBER-MANUFACTURING INDUSTRY. Available from: https://www.ncbi.nlm.nih.gov/books/NBK304412/

7. Barkan GA et al. The Paris System for Reporting Urinary Cytology: The quest to develop a standardized terminology. Acta Cytol 2016;60(3):185-97.

8. MedCalc Software Ltd. Diagnostic test evaluation calculator and multiple regression. https://www.medcalc. org/calc/diagnostic_test.php and https://www.medcalc. org/manual/multiple-regression.php (Version 20.009; accessed June 22, 2021).

9. Nurminen P, Ettala O, Seppänen M, Taimen P, Boström PJ, Kaipia A. Urine cytology is a feasible tool for assessing erythematous bladder lesions after bacille Calmette-Guérin (BCG) treatment. BJU Internat 2019; 123(2):246-51.

10. Hofland CA, Mariani AJ. Is cytology required for a hematuria evaluation? J Urol 2004;171:324-6

11. Mishriki SF, Aboumarzouk O, Vint R, Grimsley SJ, Lam T, Somani B. Routine urine cytology has no role in hematuria investigations. J Urol 2013;189:1255-8

12. Cancer in the West Midlands, Public Health England. 2017 [PDF file]. Available from https://assets.publishing.

J Endolum Endourol Vol 4(3):e1-e7; October 12, 2021.

This article is distributed under the terms of the Creative Commons Attribution-Non

Commercial 4.0 International License. (c) Moore et al. 
service.gov.uk/government/uploads/system/uploads/ attachment_data/file/639592/Cancer_in_theWest_Midlands.pdf.

13. Owens CL, Vandenbussche CJ, Burroughs FH, Rosenthal DL. A review of reporting systems and terminology for urine cytology. Cancer Cytopathol 2013;121:9-14.

14. Brimo F, Vollmer RT, Case B, Aprikian A, Kassouf W, Auger M. Accuracy of urine cytology and the significance of an atypical category. Am J Clin Pathol 2009;132:785-93.
15. Muus Ubago J, Mehta V, Wojcik EM, Barkan GA. Evaluation of atypical urine cytology progression to malignancy. Cancer Cytopathol 2013;121:387-91.

16. The Royal College of Pathologists. Meeting pathology demand: histopathology workforce census. 2018; Available at https:/www.rcpath.org/uploads/ assets/952a934d-2ec3-48c9-a8e6e00fcdca700f/ Meeting-Pathology-Demand-Histopathology-WorkforceCensus-2018.pdf (accessed June 22, 2021). 